\title{
A controlled estimate of the risk of HIV infection in Ethiopian children with tuberculosis
}

\author{
I. BERGGREN PALME ${ }^{1,3 *}$, B. GUDETTA ${ }^{2}$, H. DEGEFU ${ }^{2}$, L. MUHE ${ }^{2}$, \\ J. BRUCHFELD ${ }^{1}$ AND J. GIESECKE ${ }^{3}$ \\ ${ }^{1}$ Department of Infectious Diseases, Huddinge University Hospital, Karolinska Institutet, Stockholm, Sweden \\ ${ }^{2}$ Ethio-Swedish Children's Hospital, Department of Paediatrics, Faculty of Medicine, \\ Addis Ababa University, Addis Ababa, Ethiopia \\ ${ }^{3}$ Unit for Infectious Disease Epidemiology, Microbiological and Tumour Biology Centre, \\ Karolinska Institutet, Stockholm, Sweden
}

(Accepted 7 July 2001)

\section{SUMMARY}

To quantify the risk of human immunodeficiency virus (HIV) infection in children with tuberculosis (TB) a hospital-based, 1-year prospective, case-control study was performed in Addis Ababa, Ethiopia. Children with TB were compared to a control group of children admitted for elective surgery. The control group was also compared to a recent census of the background population. The crude odds ratio for HIV infection was $8 \cdot 6$ (95\% CI 2.2-73). After adjustment for possible confounders in a multivariate regression model, the odds ratio for HIV infection in children with TB was found to be $12 \cdot 7$ (95\% CI 2.9-55). Of several independent determinants of TB assessed in the study, this association was the strongest. Until HIV transmission has reached its peak, an increasing burden of dual infection among Ethiopian children is to be expected.

\section{INTRODUCTION}

The close association between tuberculosis (TB) and infection with human immuno-deficiency virus (HIV) is today a well-known reality. In the initial 1986 classification system for HIV infection presented by the US Centers for Disease Control, extra-pulmonary TB was one of the clinical disease criteria for AIDS [1], and pulmonary TB was included in the revised 1993 classification [2]. The great majority of patients with dual infection is found in sub-Saharan Africa, and several studies on adults have identified HIV as the major risk factor for developing clinical TB in this part of the world [3-7]. Less is known about the influence of the HIV epidemic on TB in children, but studies from Zambia, Ivory Coast and South Africa

* Author for correspondence: Unit for Infectious Disease Epidemiology, Swedish Institute for Infectious Disease Control, SE17182 Solna, Sweden. strongly suggest that HIV is a risk factor for paediatric $\mathrm{TB}$ as a result of increased susceptibility and increased exposure to infected adults [8-11]. However it has also been argued that TB-HIV associations in children are overestimated in most studies due to methodological weaknesses, such as recruitment bias (hospital based, more seriously ill, non-accurate diagnosis) [12].

In Ethiopia, TB was a public health problem even before the introduction of HIV. The spread of HIV further increased the incidence of TB, which now is the major cause of hospitalization and hospital death [13]. The HIV epidemic reached Ethiopia 5-10 years later than most other countries in sub-Saharan Africa, the first AIDS case in the country being diagnosed in 1986 [14]. The HIV epidemic has been continuously growing since then, and in 1997 the seroprevalence among pregnant women in Addis Ababa had reached $18 \%$ [15], and $57 \%$ of adult, culture-positive TB patients, recruited from the same study site as our 
study, were co-infected with HIV [16]. HIV seroprevalence among children with and without TB in Ethiopia is not known, but paediatric HIV infection has been reported [17-20].

The aim of this study was to estimate the seroprevalence of HIV in children with TB compared to the seroprevalence in a control group of children as far as possible representative of the background population. As calculation of a relative risk, or an odds ratio (OR), for HIV infection between two such groups might easily be confounded by differences in age distribution, socio-economic background, range of possible exposures to HIV, etc. we decided to include data on such factors in the study. We also assessed the degree of bias introduced by using selected hospital-based controls as representatives of the child population in Addis Ababa.

\section{METHODS}

\section{Study setting}

In 1996, Addis Ababa had an estimated population of $2 \cdot 1$ million, $32 \%$ of whom were under the age of 15 [21]. The city is divided into 28 administrative areas called woredas. The study was performed at the EthioSwedish Children's Hospital (ESCH), the main paediatric hospital in the city. Children with suspected TB are sent to the TB clinic for diagnosis and treatment except for the critically ill who are admitted directly to the wards. Based on a pilot survey in 1995 , we estimated that of all children diagnosed with clinical TB within the National Health Service (NHS) in Addis Ababa, around 50\% would be seen at the TB clinic at $\mathrm{ESCH}$ [22]. This makes ESCH the major centre for paediatric TB treatment in the city, as well as in the country. We have no information on children with TB not diagnosed within the NHS, who may have received treatment elsewhere or remained undiagnosed. The surgery department of the ESCH is the only centre for paediatric elective surgery in the city.

\section{Study population}

Case definition

A child, 0-14 years of age, with residency in Addis Ababa, presenting at the ESCH with a first episode of clinical TB between 11 December 1995 and 28 January 1997.

\section{Control definition}

A child in the same age group, resident in Addis Ababa coming for elective surgery to ESCH during the same study period. We expected this group to include younger age groups, which was considered acceptable in view of the fact that most HIV-infected children were expected to be found in this age group. We also expected a male predominance, which we deemed not likely to affect results, there being no known sex-associated differences in children with the studied conditions. The main advantage of this choice of control group was its being recruited from all layers of society and in generally good health except for the underlying condition, mostly congenital or posttraumatic. In order to make certain that the controls did not have TB, children with signs of lower respiratory infection or other clinical signs or symptoms of TB were excluded.

\section{Recruitment of patients and data collection}

After informed consent by the responsible caretaker of the child, study subjects were consecutively included as they appeared at the hospital. Cases and controls were evaluated at enrolment by one of the two physicians responsible for the TB unit at $\mathrm{ESCH}$ (B.G., H.D.) and clinical and laboratory data were recorded on a form prepared for the study. Sputa and/or gastric aspirates were collected from cases with pulmonary manifestations. HIV testing of cases was accompanied by pre- and post-test counselling by the treating physician. HIV testing of controls was done anonymously, but a separate individual test was offered outside the study, if requested by the parents. Socio-economic and medical background data were collected for both groups through a structured interview with the caretaker of the child, using a pretested questionnaire. All interviews were performed by the same research nurse.

\section{The 1994 Population and Housing Census of Ethiopia; Addis Ababa}

In order to ascertain that the selection of controls did not differ substantially from the background population we compared basic demographics to the results of the 1994 Population and Housing Census [21], which was the second national population and housing census to be conducted in Ethiopia. It was conducted by enumerators who went from house to 
house filling in questionnaires designed for the purpose. Information on number of household members, their ethnic background, and their sex and age composition was collected from all households covered by the census. More detailed information on education, economic activity and housing conditions was collected from one out of five households using a systematic sampling selection.

\section{Criteria for TB diagnosis}

The diagnosis of TB was based on an adapted version of the WHO guidelines for the diagnosis of pulmonary TB in children published in 1983 and routinely used at the hospital [23]. TB was diagnosed by the presence of two or more of the following criteria: (a) symptom complex suggestive of TB (fever more than 2 weeks, cough more than two weeks, night sweats, weight loss); (b) household contact with proved or highly suggestive case of TB; (c) suggestive chest X-ray findings (primary complex, hilar lymphadenopathy, cavity, miliary pattern, effusions or any other infiltration compatible with TB); (d) identification of mycobacteria from sputum and/or gastric aspirate by staining for acid-fast bacilli or by culture; (e) positive Mantoux test, i.e. an area of induration of $10 \mathrm{~mm}$ or more, 48-72 h after injection of PPD (purified protein derivate); (f) pathological findings compatible with tuberculosis from cerebro-spinal fluid, fine needle aspiration, biopsy or other surgically removed lesion.

The TB cases were classified either as single focus or disseminated. Patients with a single focus were further subdivided into pulmonary or extra-pulmonary. Those classified as disseminated TB may or may not have had pulmonary involvement.

\section{TB culture}

The sputum and gastric aspirate samples were inoculated onto three Löwenstein-Jensen culture tubes and the inoculated media were incubated at $37^{\circ} \mathrm{C}$. Species identification was performed using standard biochemical tests [24]. TB culture was performed at Armauer Hansen Research Institute (AHRI), Addis Ababa.

\section{Criteria for HIV diagnosis}

Serum samples from all patients were tested by two enzyme-linked immunosorbent assays (EIA) Enzygnost (Enzygnost Anti-HIV1/2Plus, Behring- werke, Marburg, Germany) and Wellcozyme (Wellcome Diagnostics, Dartford, UK). Patients older than 18 months with positive results on both EIAs were considered as HIV-1 antibody positive and HIV infected. Discordant or borderline tests were confirmed by commercially available HIV-1 Western blot (Diagnostic Biotechnology Ltd, Singapore). If negative in the confirmatory test, the sample was considered negative. Younger children may carry HIV antibodies of their mothers for up to 18 months. For this reason, sera positive or borderline for HIV antibodies in children less than 19 months were examined for the presence of HIV antigen using a modified HIV-1 p24 antigen assay [25]. To confirm the presence of HIV-1 antigen all samples were neutralized with antibodies. If negative in the p24 antigen tests, the child was considered uninfected. The EIA tests were performed at AHRI, Addis Ababa. Western blot and HIV-1 p24 antigen assays were performed at the Department of Virology, Swedish Institute for Infectious Disease Control, Stockholm (SMI). HIV-2 has to our knowledge not been reported in Ethiopia.

\section{Statistical analyses}

Questionnaire design, data entry and statistical analyses, except for the analyses stated below, were based on version 6.04 of the Epi-Info software (Centers for Disease Control and Prevention, Atlanta, Georgia). Differences in proportions between cases and controls regarding socio-economic variables and possible risk factors for tuberculosis were assessed with $\chi^{2}$ or Fisher's exact test. Stata statistical Software: Release 6.0 (Stata Corp. 1999, College Station, Texas, USA) was used to perform the Wilcoxon rank-sum test to determine the difference in level of income between cases and controls. OR and $95 \%$ CI were calculated to measure the strength of association between the selected covariates and TB disease. Factors found to be significantly $(P<0.05)$ associated with tuberculosis were added step-wise into a multiple logistic regression model using the programme package SPSS (SPSS Inc., Chicago, IL, USA). Because of its skewed distribution, logarithmic transformation of income was used in this model.

\section{Ethical approval}

The study was approved by the Ethical Committees of the Karolinska Institutet, Sweden, of the Medical 
Table 1. Characteristics of control population (Ethio-Swedish Children's hospital 1995-7) compared to background population (1994 Population and Housing Census Ethiopia; results for Addis Ababa)

\begin{tabular}{|c|c|c|c|c|}
\hline & \multicolumn{2}{|l|}{$\begin{array}{l}\text { Control } \\
(n=122)\end{array}$} & \multirow{2}{*}{$\begin{array}{l}\text { Census } \\
\text { Population }=2 \cdot 2 \text { million } \\
\text { Children }=714872 \\
\text { No. }(\%)\end{array}$} & \multirow[b]{2}{*}{$P$-value } \\
\hline & No. $(\%)$ & $\left(95 \% \mathrm{CI}^{*}\right)$ & & \\
\hline \multicolumn{5}{|c|}{ Child characteristics $\dagger$} \\
\hline Female & $47(39)$ & $(20-48)$ & $365055(51)$ & $<0 \cdot 01$ \\
\hline Age $<5$ & $62(51)$ & $(42-60)$ & $182148(26)$ & $<0.001$ \\
\hline \multicolumn{5}{|l|}{ Ethnic } \\
\hline Amhara & $52(43)$ & $(34-52)$ & $343138(48)$ & n.s. \\
\hline Gurague & $27(22)$ & $(15-31)$ & $125103(18)$ & n.s. \\
\hline Oromo & $25(20)$ & $(14-29)$ & $135826(19)$ & n.s. \\
\hline Other & $18(15)$ & $(6-18)$ & $110805(15)$ & n.s. \\
\hline \multicolumn{5}{|c|}{ Characteristics of households $\dagger$} \\
\hline Illiterate father & $15(13)$ & $(7-19)$ & (11) & n.s. \\
\hline Illiterate mother & $25(21)$ & $(14-29)$ & (24) & n.s. \\
\hline Mud house & $101(83)$ & $(75-89)$ & (83) & n.s. \\
\hline Piped water & $122(100)$ & - & (97) & n.s. \\
\hline Electricity & $121(99)$ & $(95-100)$ & (96) & n.s. \\
\hline Telephone & $20(16)$ & $(10-24)$ & (18) & n.s. \\
\hline Radio & $100(82)$ & $(74-88)$ & (70) & $<0.01$ \\
\hline Television & $28(23)$ & $(16-31)$ & (16) & $0 \cdot 04$ \\
\hline
\end{tabular}

* $\mathrm{CI}=$ confidence interval for percentage.

$\dagger$ In the census child characteristics are based on individuals $<15$ years, while household characteristics are based on $1 / 5$ households.

Faculty, Addis Ababa University and by the Ethiopian Science and Technology Commission, Ethiopia.

\section{RESULTS}

Over the 13-month study period, 377 children with clinical TB were enrolled, 340 from the TB clinic and 37 from the wards. This includes all eligible children at the TB clinic and $80-90 \%$ of eligible children at the wards. One hundred and eighty-five (49\%) were diagnosed as having pulmonary TB, $77(20 \%)$ as having extra-pulmonary TB and $115(31 \%)$ as having disseminated TB. A positive TB culture was obtained from $131(44 \%)$ of the 297 children who had pulmonary $\mathrm{TB}$ or disseminated $\mathrm{TB}$ with pulmonary manifestations. Age ranged from 6 months to 14 years, with a median of 6.5, and $186(49 \%)$ were boys.

During the same period 122 consecutive non-TB patients were included from the surgery department as controls. Their ages ranged from 4 months to 15 years with a median of 4 years. Seventy-five $(61 \%)$ were boys. The most common diagnoses were congenital deformities $(27 \%)$, undescended testis/hernia/ hydrocoele $(17 \%)$, and fractures/post-burn contractures $(11 \%)$.

\section{Comparison of characteristics between controls and the 1994 Addis Ababa census}

According to the census the overall population of children under the age of 15 years is smaller in Addis Ababa (32\%) compared to the country as a whole $(45 \%)$. As expected, children in the control group were younger and included a higher proportion of boys than the census. Other characteristics like ethnicity, markers for education and socio-economic status were similar (Table 1).

\section{Analysis of possible confounders associated with HIV seropositivity}

The geographical distribution of the patients is shown in Figure 1, from which it can be seen that a higher proportion of cases than of controls came from woredas close to the hospital. In Table 2 , socioeconomic determinants and exposures to interventions related to possible transmission of HIV are compared between the two groups. TB patients had the same distribution of girls and boys while the control patients had a higher proportion of boys. The age distribution differed in that TB patients were evenly distributed 

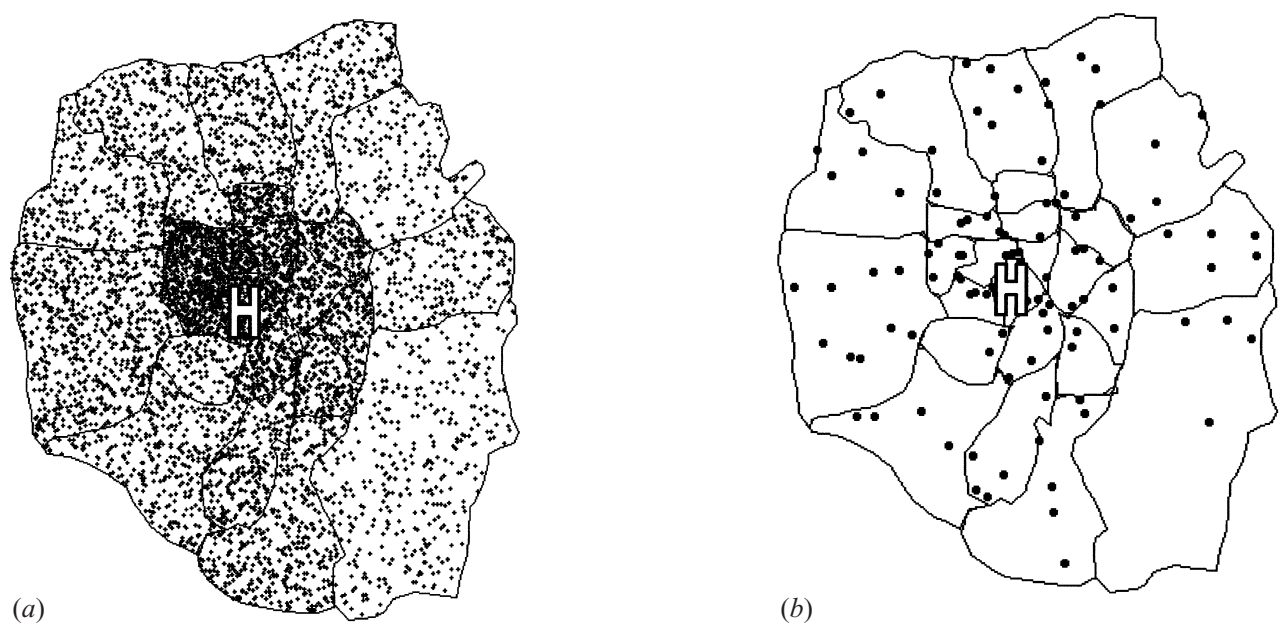

(b)
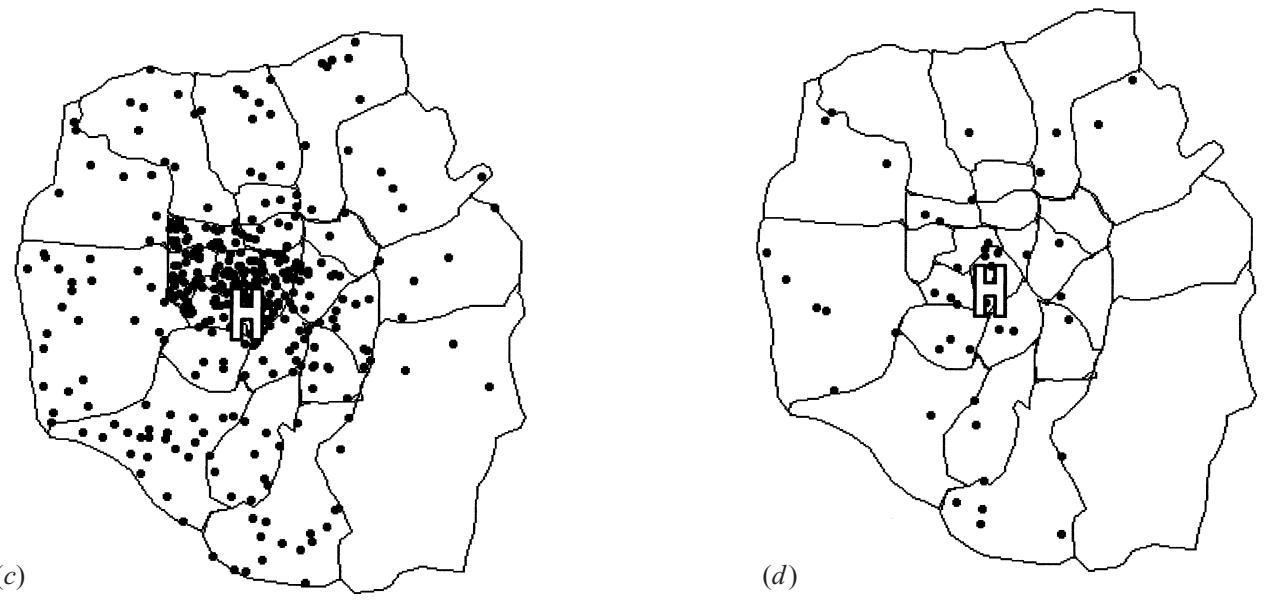

Fig. 1. Distribution within Addis Ababa of $(a)$ total child population ( 1 dot $=100$ children $),(b)$ control patients $(1 \mathrm{dot}=$ 1 child), (c) TB patients ( 1 dot $=1$ child), and $(d)$ TB patients co-infected with HIV ( 1 dot $=1$ child). Area dot map; lines outline woredas. Ethio-Swedish Children's Hospital is marked ' $\mathrm{H}$ ' on the maps. Maps were drawn with the EpiMap module of EpiInfo, version 6.04.

over the age groups $<5$ years $(34 \%)$, 5-9 years $(40 \%)$ and $>9$ years $(26 \%)$, while in the control group $50 \%$ were $<5$ years old. The four major ethnic groups: Oromo, Amhara, Gurague and Tigray were all represented, with a slight difference in distribution between cases and controls, the Amharas being less and the Guragues more represented among the TB patients. The illiteracy rate among parents of the TB patients was almost double that of controls. Although no major differences in living conditions or ownership of luxury items between cases and controls could be seen, all proxy measures of socio-economic status point to a less favourable situation among the TB patients. Blood transfusion and other interventions carrying a risk of HIV transmission were evenly distributed between cases and controls except for uvulectomy, which had more often been performed on TB patients. Uvulectomy is a traditional operation, common in Ethiopia, believed to protect the small child from suffocation during pharyngitis. Immunization coverage was higher in the control group for all routine vaccines: Bacillus Calmette-Guerin (BCG), polio, measles and diphtheria/pertussis/tetanus (DPT).

\section{HIV prevalence in cases and controls}

All 377 children with tuberculosis included in the study were tested for HIV antibody and 12.5\% $(47 / 377)$ were found to be positive. Forty-six of the 377 children were less than 19 months. Of these, $33 \%$ $(15 / 46)$ were positive in ELISA and they were also 
Table 2. Association between tuberculosis, HIV infection and possible confounders in children from Addis Ababa, Ethiopia

\begin{tabular}{|c|c|c|c|c|}
\hline & $\begin{array}{l}\text { TB patients } \\
(n=377)\end{array}$ & $\begin{array}{l}\text { Controls } \\
(n=122)\end{array}$ & $\begin{array}{l}\text { Odds ratio } \\
(95 \% \mathrm{CI})\end{array}$ & $P$-value \\
\hline \multicolumn{5}{|l|}{ Child characteristics (\%) } \\
\hline HIV infection & $12 \cdot 5$ & 2 & $8 \cdot 6(2 \cdot 2,74)$ & $<0 \cdot 001 *$ \\
\hline Female & 51 & 39 & $1 \cdot 4(1 \cdot 1,2 \cdot 5)$ & $0 \cdot 02$ \\
\hline Mean age (months \pm S.D.) & $79 \pm 44$ & $64 \pm 46$ & & $<0 \cdot 001$ \\
\hline \multicolumn{5}{|l|}{ Ethnic } \\
\hline Amhara & 31 & 43 & $0 \cdot 6(0 \cdot 4,1 \cdot 8)$ & $0 \cdot 02$ \\
\hline Gurague & 33 & 21 & $1 \cdot 7(1 \cdot 0,2 \cdot 8)$ & $0 \cdot 04$ \\
\hline Oromo & 25 & 21 & $1 \cdot 3(0 \cdot 8,2 \cdot 2)$ & n.s. $\dagger$ \\
\hline Tigray & 10 & 11 & $0 \cdot 9(0 \cdot 4,1 \cdot 8)$ & n.s. \\
\hline Other & 2 & 4 & & \\
\hline \multicolumn{5}{|l|}{ Medical history of ( $\%)$} \\
\hline Blood Transfusion & 2 & 3 & $0 \cdot 6(0 \cdot 1,2 \cdot 6)$ & n.s. \\
\hline Circumcision & 51 & 45 & $1 \cdot 3(0 \cdot 8,2 \cdot 0)$ & n.s. \\
\hline Uvulectomy & 49 & 41 & $1 \cdot 9(1 \cdot 2,3 \cdot 0)$ & $0 \cdot 01$ \\
\hline Three doses of DPT & 71 & 91 & $0 \cdot 2(0 \cdot 1,0 \cdot 5)$ & $<0 \cdot 001$ \\
\hline BCG scar & 40 & 64 & $0 \cdot 4(0 \cdot 2,0 \cdot 6)$ & $<0.001$ \\
\hline \multicolumn{5}{|l|}{ Characteristics of households (\%) } \\
\hline Illiterate father & 21 & 13 & $1 \cdot 9(1 \cdot 1,3 \cdot 5)$ & $0 \cdot 04$ \\
\hline Illiterate mother & 39 & 21 & $2 \cdot 5(1 \cdot 6,4 \cdot 1)$ & $<0 \cdot 001$ \\
\hline Mud house & 84 & 83 & $1 \cdot 1(0 \cdot 6,1 \cdot 9)$ & n.s. \\
\hline Electricity & 97 & 99 & $0 \cdot 3(0 \cdot 01,1 \cdot 9)$ & n.s. \\
\hline Radio & 71 & 82 & $0 \cdot 5(0 \cdot 3,0 \cdot 9)$ & $0 \cdot 02$ \\
\hline Telephone & 10 & 16 & $0 \cdot 6(0 \cdot 3,1 \cdot 1)$ & $0 \cdot 07$ \\
\hline \multicolumn{5}{|l|}{ Monthly income (E Birr) $\ddagger$} \\
\hline Median & 200 & 300 & & $<0 \cdot 01 \S$ \\
\hline Lower quartile & 100 & 120 & & \\
\hline Upper quartile & 400 & 592 & & \\
\hline Mean persons per room \pm s.D. & $3 \cdot 4 \pm 1 \cdot 8$ & $2 \cdot 8 \pm 1 \cdot 4$ & & $0 \cdot 02$ \\
\hline
\end{tabular}

* Fisher's exact test. $\dagger$ n.s., not significant $(P>0 \cdot 05)$.

$\$ 1 \mathrm{US} \$=6$ E Birr. $\S$ Wilcoxon rank-sum test.

positive in HD-p24ag test and Western Blot. Three children in this age group had a borderline result in ELISA but were negative in HD-p24 and thus considered negative. Among children with a positive TB culture from sputum or gastric aspirate, HIV prevalence was $7 \cdot 6 \%(10 / 131)$. Children with disseminated forms of TB had an HIV seroprevalence of $21 \%(24 / 115)$. All HIV infected children were found in the age group less than 10 years. Figure 2 shows prevalence of HIV infection in different age groups combined with different manifestations of TB.

In the control group, prevalence of HIV-1 infection was $1.6 \%(2 / 122)$. The two HIV antibody positive children in the control group were 5 months and 4 years old, respectively.

The crude OR for being co-infected with HIV in children with TB was thus $8 \cdot 6$ (exact $95 \% \mathrm{CI}: 2 \cdot 2-73$ ). HIV status was entered together with all covariates from Table 2 carrying a univariate $P$-value of less than
0.05 into a multivariate analysis using logistic regression. This resulted in an increase in the adjusted OR (AOR) to 12.7 (95\% CI 2.9-55). Other determinants independently associated with tuberculosis were: higher age, absence of BCG scar, less than three doses of DPT, and low income (Table 3).

\section{DISCUSSION}

Ethiopia is estimated to have more than $140000 \mathrm{HIV}$ infected children and the largest population of children with AIDS in the world [26]. In this study the overall prevalence of HIV infection in children from Addis Ababa diagnosed with TB was $12.5 \%$. In children with culture-confirmed TB, $7 \cdot 6 \%$ were HIV infected, which can be seen as a minimum figure, since among children with disseminated forms of TB a much higher seroprevalence was found $(21 \%)$. As 


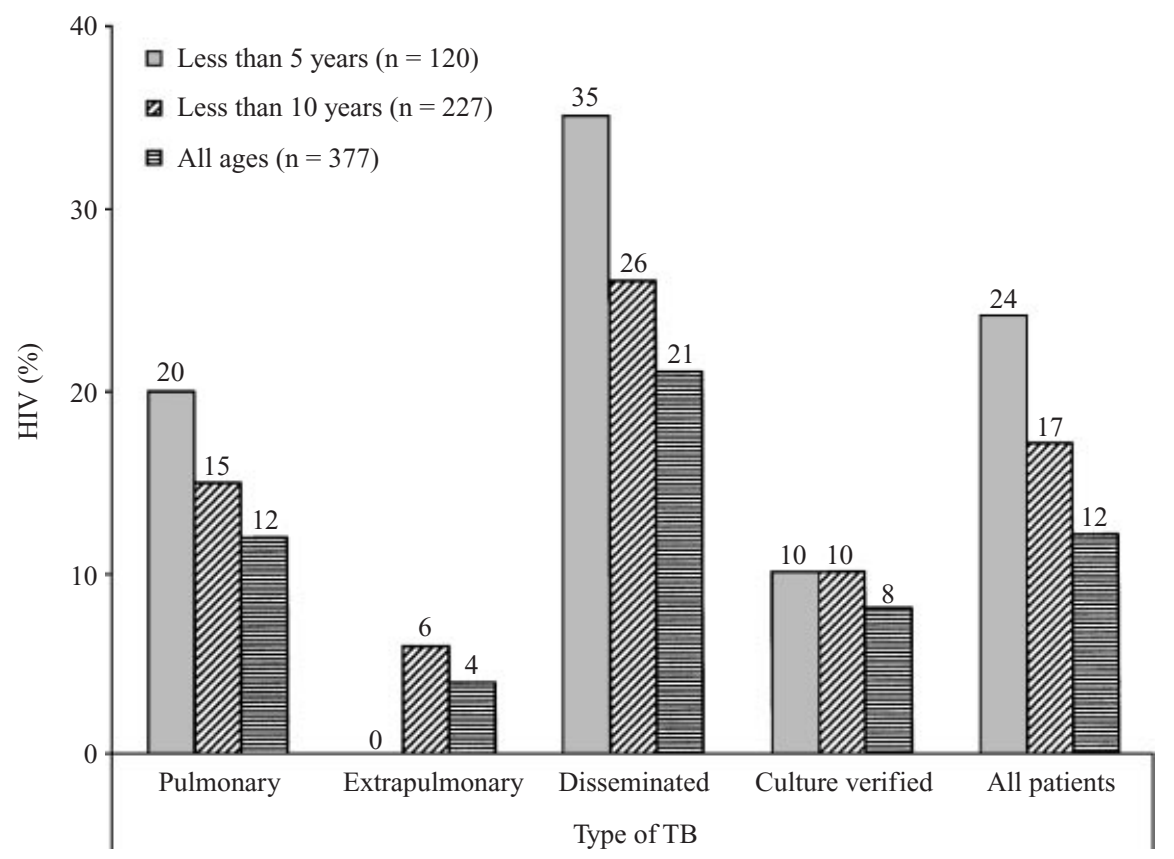

Fig. 2. HIV prevalence related to clinical presentation and age in children with tuberculosis.

Table 3. Factors independently associated with risk of tuberculosis in Addis Ababa children

\begin{tabular}{lccll}
\hline \hline & $\begin{array}{l}\text { TB patients } \\
(n=377)\end{array}$ & $\begin{array}{l}\text { Controls } \\
(n=122)\end{array}$ & $\begin{array}{l}\text { Adjusted odds ratio } \\
(95 \% \mathrm{CI})\end{array}$ & $P$-value \\
\hline Age in months (mean \pm s.D. $)$ & $79 \pm 44$ & $64 \pm 46$ & $1 \cdot 009(1 \cdot 004,1 \cdot 015)$ & $0 \cdot 001$ \\
Monthly income (median) & 200 & 300 & $0 \cdot 7(0 \cdot 5,0 \cdot 9)^{*}$ & $0 \cdot 003$ \\
DPTIII & 268 & 112 & $0 \cdot 3(0 \cdot 1,0 \cdot 8)$ & $0 \cdot 01$ \\
BCG scar & 151 & 78 & $0 \cdot 5(0 \cdot 3,0 \cdot 8)$ & $0 \cdot 002$ \\
HIV & 47 & 2 & $12 \cdot 7(2 \cdot 9,55)$ & $0 \cdot 001$ \\
\hline \hline
\end{tabular}

* Odds ratio calculated on logarithm of monthly income.

expected, HIV co-infection was restricted to the younger ages. The seroprevalence of $17 \%$ in children less than 10 years is comparable to $19 \%$ found in Abidjan 1994/5 in the same category of patients [10]. A study of hospitalized children with TB in Johannesburg 1996 [11], found an HIV seroprevalence of $42 \%$, thus much higher than our finding. If, however, we restrict our analysis to hospitalized children only, we found a seroprevalence in Addis Ababa of $35 \%$.

Drawing conclusions from a hospital-based study to the general population is often difficult because of selection bias. In this study we were restricted to the hospital for finding both TB patients and controls. However, TB patients coming to $\mathrm{ESCH}$ represent around $50 \%$ of diagnosed paediatric TB cases in the city and included both severe (wards) and milder forms (TB clinic). The selected control group was considered to better represent the general population as regards risk of HIV infection than other non-TB patients attending the hospital. Comparison with the 1994 census also showed that, besides those differences in age distribution and gender, which were expected and controlled for in the analysis, no major discrepancies in the variables examined were found. A serological study based on sera from a household survey 2 years before our study found 3 HIV positives in 242 blood samples from children aged $0-4$ years [20], which is similar to our control group.

The unadjusted OR of HIV seroprevalence in cases to controls was almost 9. However, as in the earlier referred study in Abidjan [10] our study also looked at several other factors associated with TB. Our TB patients came from families with less formal education, less income and lived in more crowded conditions than the average non-TB family. This is 
similar to the findings in Abidjan. In addition, our control patients lived in a geographically more scattered area and were more often vaccinated than children with TB. As can be seen from Table 3, of all the determinants found independently associated with TB, HIV infection showed by far the strongest association. Some possible biases could not be controlled for. Thus, as cases and controls did not come from exactly the same area (Fig. 1), and since HIV infection may not be evenly distributed within the city, there is a possibility of selection bias that could influence the difference between cases and controls in either direction.

Lack of BCG and not having completed three doses of DPT both remained significant confounders in the multivariate analysis. The role of BCG and TB is debated, but there is strong consensus on its protective effect against disseminated disease in children [27]. In our study controls had a BCG scar twice as often as children with $\mathrm{TB}$, indicating a $50 \%$ vaccine efficacy in this setting. However, since prevalence of DPT vaccination was also lower among cases independently of $\mathrm{BCG}$, the association of TB and lack of vaccination could be explained by residual confounding linked to education and socio-economic status.

In spite of these limitations we believe that this study to gives a realistic picture of the HIV prevalence among children with TB and that the controls reflect the HIV situation in the general child population of Addis Ababa during the study period. Assuming this is true, we may conclude that children with TB have an almost 13-fold risk of being HIV infected compared to the background population. The findings confirm the association between TB and HIV infection in children but also demonstrates that the magnitude of this association is strongly linked to the selection of study population as was pointed out by Coovadia et al. [12].

An alarming increase of HIV seroprevalence over time in paediatric TB patients has been reported from Lusaka, Zambia (24\% in 1989 to $69 \%$ in 1992) and from Abidjan, Ivory Coast (12\% in 1989/90 to 19\% in 1996) [8-10]. Considering that the HIV epidemic reached Ethiopia late and, according to data up to 1998 , tends to follow an epidemic curve similar to that of the Ivory Coast, it is to be expected that the proportion of the paediatric population with $\mathrm{TB}$, coinfected with HIV, will increase also in Addis Ababa. Dual infection threatens to become a major cause of morbidity and mortality not only among adults but also among Ethiopian children.

\section{ACKNOWLEDGEMENTS}

This study was a collaboration between the Faculty of Medicine at Addis Ababa University, the Armauer Hansen Research Institute, Addis Ababa and the Karolinska Institutet, Stockholm. It was financially supported by the Norwegian and the Swedish Save the Children Foundation. We thank all the patients, their parents and the staff at the $\mathrm{ESCH}$; nurse Aregesh Aragie (ESCH) for her efficient and careful handling of patients and records; research assistants Abebesh Demissie (AHRI), Selamawit Tadesse (AHRI) for careful assistance with HIV-serology and mycobacterial culture, Alemayo Kifle (AHRI) for facilitating the proper handling of biological samples and Johan Lindbäck (SMI) for statistical assistance.

\section{REFERENCES}

1. Centers for Disease Control. Revision of the CDC surveillance case definition for acquired immunodeficiency syndrome. MMWR 1987; 361: S1-15.

2. Centers for Disease Control and Prevention. 1993 revised classification system for HIV infection and expanded surveillance case definition for AIDS among adolescents and adults. MMWR 1992; 41 (RR1-17):

3. Standaert B, Niragira F, Kadende P, Piot P. The association of tuberculosis and HIV infection in Burundi. AIDS Res Hum Retroviruses 1989; 5 : 247-51.

4. Colebunders RL, Ryder RW, Nzilambi N, et al. HIV infection in patients with tuberculosis in Kinshasa, Zaire. Am Rev Respir Dis 1989; 139: 1082-5.

5. De Cock KM, Soro B, Coulibaly IM, Lucas SB. Tuberculosis and HIV infection in sub-Saharan Africa. JAMA 1992; 268: 1581-7.

6. Houston S, Ray S, Mahari M, et al. The association of tuberculosis and HIV infection in Harare, Zimbabwe. Tuber Lung Dis 1994; 75: 220-6.

7. Styblo K. The impact of HIV infection on the global epidemiology of tuberculosis. Bull Int Union Tuberc Lung Dis 1991; 66: 27-32.

8. Luo C, Chintu C, Bhat $\mathrm{G}$, et al. Human immunodeficiency virus type-1 infection in Zambian children with tuberculosis: changing seroprevalence and evaluation of a thiacetazone-free regimen. Tuber Lung Dis 1994; 75: 110-5.

9. Sassan-Morokro M, De Cock KM, Ackah A, et al. Tuberculosis and HIV infection in children in Abidjan, Côte d'Ivoire. Trans R Soc Trop Med Hyg 1994; 88: 178-81.

10. Mukadi YD, Wiktor SZ, Coulibaly IM, et al. Impact of HIV infection on the development, clinical presentation, and outcome of tuberculosis among children in Abidjan, Côte d'Ivoire. AIDS 1997; 11: 1151-8.

11. Madhi SA, Huebner RE, Doedens L, Aduc T, Wesley D, Cooper PA. HIV-1 co-infection in children 
hospitalised with tuberculosis in South Africa. Int $\mathbf{J}$ Tuberc Lung Dis $2000 ; 4$ : 448-54.

12. Coovadia HM, Jeena P, Wilkinson D. Childhood human immunodeficiency virus and tuberculosis coinfections: reconciling conflicting data. Int $\mathbf{J}$ Tuberc Lung Dis 1998 ; 2: 844-51.

13. Guidelines for the National Tuberculosis Control Programme in Ethiopia, August 1992.

14. Lester FT, Ayehunie S, Zewdie D. Acquired immunodeficiency syndrome: seven cases in Addis Ababa hospital. Ethiop Med J 1988; 26: 139-45.

15. AIDS in Ethiopia Epidemiology and AIDS Department, Ministry of Health, 2nd ed, 1998.

16. Bruchfeld J, Aderaye G, Berggren Palme I, Bjorvatn B, Källenius G, Lindquist L. Sputum concentration improves diagnosis of tuberculosis in a setting with a high prevalence of HIV. Trans R Soc Trop Med Hyg 2000; 94: 677-80.

17. Beyene $\mathrm{H}$, Moss W. Clinical and epidemiological features of HIV-1 seropositive hospitalised Ethiopian children. Ethiop Med J 1991; 29: 57-61.

18. Bedri A, Kebede S, Negassa H. Sociodemographic profile of children affected by AIDS in Addis Ababa. Ethiop Med J 1995; 33: 227-34.

19. Muhe L. A four-year cohort study of HIV seropositive Ethiopian infants and children: clinical course and disease patterns. Ethiop Med J 1997; 35: 103-15.

20. Fontanet AL, Messele T, Dejene A, et al. Age- and sex- specific HIV-1 prevalence in the urban community setting of Addis Ababa, Ethiopia. AIDS 1998; 12: 315-22.

21. Central Statistical Authority. The 1994 Population and Housing Census of Ethiopia. Results for Addis Ababa. Addis Ababa, Ethiopia, 1995.

22. Berggren Palme I. Co-operative project on tuberculosis/HIV interactions in Ethiopia. Internal report to Swedish Agency for Research Co-operation with Developing Countries (SAREC), 1995.

23. World Health Organisation. Provisional guidelines for the diagnosis and classification of the EPI target diseases for primary health care, surveillance and special studies. EPI/GEN/83/4, 1983.

24. Kent, PT, Kubica GP. Public health mycobacteriology. A guide for level III laboratory. Atlanta; CDC, 1985.

25. Lyamuya E, Bredberg-Rådén U, Massawe A, et al. Performance of a modified HIV-1 p24 antigen assay for early diagnosis of HIV-1 infection in infants and prediction of mother-to infant transmission of HIV-1 in Dar es Salaam, Tanzania J. Acquir Immune Defic Syndr 1996; 12: 421-6.

26. UNAIDS Report on the global HIV/AIDS epidemic June 2000. UNAIDS ISBN 92-9173-000-9.

27. Colditz GA, Berkey CS, Mosteller F, et al. The efficacy of Bacillus Calmette Guerin vaccination of newborns and infants in the prevention of tuberculosis: metaanalysis of the published literature. Pediatrics 1995; 96: 29-35. 